\title{
Scaling limit of weakly bound triatomic states
}

\author{
T. Frederico, ${ }^{1}$ Lauro Tomio, ${ }^{2}$ A. Delfino, ${ }^{3}$ and A. E. A. Amorim ${ }^{4}$ \\ ${ }^{1}$ Departamento de Física, Instituto Tecnológico de Aeronáutica, Centro Técnico Aeroespacial, 12228-900 São José dos Campos, Brazil \\ ${ }^{2}$ Instituto de Física Teórica, Universidade Estadual Paulista, 01405-900 São Paulo, Brazil \\ ${ }^{3}$ Instituto de Física, Universidade Federal Fluminense, 24210-900 Niterói, RJ, Brazil \\ ${ }^{4}$ Faculdade de Tecnologia de Jahu, CEETEPS, Jahu, Brazil \\ (Received 8 April 1998; revised manuscript received 10 August 1998)
}

\begin{abstract}
The usefulness of a scale-independent approach to identify Efimov states in three-body systems is shown by comparing such an approach with a realistic calculation in the case of three helium atoms. We show that the scaling limit is realized in practice in this case, and suggest its application to study other similar systems, including the case where two kinds of atoms are mixed. We also consider the observed large scattering length of the ${ }^{87} \mathrm{Rb}$ dimer to estimate the critical value of the ground-state energy of the corresponding trimer $(\geqslant 1.5 \mathrm{mK})$, in order to allow for one Efimov state above the ground state. [S1050-2947(99)50407-1]

PACS number(s): 34.10.+x, 05.30.Jp, 21.45.+v, 36.40.-c
\end{abstract}

Loosely bound two- and three-body systems are characterized by the fact that their sizes are much larger than the characteristic range of the two-body interaction. The best illustration for such a system is the experimentally found ${ }^{4} \mathrm{He}-{ }^{4} \mathrm{He}$ dimer with a size of about $50 \AA[1,2]$. Qualitatively, the ${ }^{4} \mathrm{He}-{ }^{4} \mathrm{He}$ interaction has roughly a depth of $11 \mathrm{~K}$ with a scattering length $\left(a_{H e}\right)$ around $100 \AA$. In such a case, approaching the limit where the two-boson energy is zero, the three-boson system presents an increasing number of loosely bound three-body states, known as Efimov states [3]. They have large spatial extension and do not depend on the details of the interaction. Although diffraction experiments [2] indicated the existence of a helium trimer, the connection with the Efimov effect is not clear. The Efimov states occur at extremely low energies and, if they exist, they are certainly related to low-temperature effects that occur in atomic systems, such as Bose condensation and superfluidity. A weakly bound triatomic state becomes important from the perspective of an ultracold gas, as it provides a repulsive three-body pseudopotential, independent of the sign of the two-atom scattering length, which could stabilize the condensate even in the case of negative scattering length $[4,5]$. Also, the three-body recombination of ultracold atoms to a weakly bound $s$ level in the Efimov limit goes to infinity [6].

In the limit of zero-range interaction, with the two-boson scattering length fixed, the three-boson ground state collapses and an infinite number of strongly bound states appears (Thomas effect [7]). The Efimov and Thomas states are related by a scale transformation and are governed by the ratio between the scattering length and the range of the force $[8,9]$. As is also well known, even when the two-body interaction is not strong enough to bind the two-body system, it can bind the corresponding three-body system. This implies that a change in sign of the two-body scattering length does not rule out the existence of Efimov states. Recently, the relation was also discussed between an effect that occurs when two identical scatterers are close together (known as proximity resonances) and the Thomas and Efimov effects [10].

Considering the possibility of atomic bulk matter, with two different species of atoms ( $\alpha$ and $\beta$ ), we study the three- body systems $\alpha-\alpha-\beta$. The $\alpha-\beta$ and $\alpha-\alpha$ systems may or may not be bound; thus the atom-atom scattering length attains a range of values. In principle, this can be realized in experiments where, by means of light or induced magnetic fields, the excited atoms have the two-atom interaction changed [11].

Two helium atoms have a very shallow and short-ranged interaction potential, with the binding energy very close to zero [1,2]; thus many aspects of the relevant three-body physics are analogous to those that have been studied in nuclear physics at very low energies [3,12-14,9]. The large scattering length compared to the range of the potential justifies the search for Efimov states in a helium-trimer system. Several theoretical studies have already considered the possibility of Efimov states in the helium trimer [2,15-21]. At first sight, one notices that the ground state is extremely sensitive to the potential-well region and also that the Efimov states are sensitive to certain features of the longe-range potential. In the following, we definitely clarify that such apparent independent sensitivities, of the ground and Efimov state energies, disappear once the dimer binding energy is kept fixed. The conclusion is that other details (knowing the dimer and trimer ground-state energies) presented in the realistic interactions that have been used are quite irrelevant to the existence of Efimov states. These features are represented in a universal scaling function, relating the trimer ground state, the dimer, and the Efimov state binding energies, which contains most of the relevant physics of realistic models.

The main purpose of the present work is to derive the scaling function, which is obtained in the scaling limit [9] when the range of the interaction goes to zero. The existence of the scaling limit is a consequence of the renormalizability of quantum mechanics with zero-range interactions [9,22]. We will show that in practice such a limit is approached by the excited state of the helium trimer, obtained in realistic calculations [19-21], clarifying the theoretical interpretation of those excited states as Efimov states. We believe that the scaling limit, once its wide application in low-energy threeparticle physics (from nuclear to molecular systems) is 
proved, must be very useful in analyzing any other similar systems (such as the rubidium systems [23,24], for example).

The scaling limit of weakly bound triatomic systems is found from the renormalization procedure of the zero-range (RZR) three-body model, where the physical inputs are twobody and three-body ground-state energies [22]. The threebody ground state energy, if not available from experiments, can be determined using a realistic two-body interaction. Even when a realistic calculation has already been used for the ground-state, one should still think about the advantages of using the RZR model to obtain other low-energy threebody observables, considering the extensive numerical task involved in a realistic calculation (see the appendix of Ref. [19]).

With the only purpose being to gain some qualitative insight into the scaling limit, let us discuss how the scaling function is justified in the context of the two-body model for a three-body system of Ref. [13], where it is considered as two heavy particles (label $\alpha$ and mass $M_{\alpha}$ ), separated by $R$, and a light one (label $\beta$ ). Subtracting the two-body binding energy $B_{\alpha \beta}$, the effective interaction between the two heavy particles, generated by the solution of the equation for lightheavy particle systems, is given by

$$
V(R)=-\frac{1}{\nu}\left[\frac{2}{a_{\alpha \beta}} \frac{e^{-R / a_{\alpha \beta}}}{R}+\frac{e^{-2 R / a_{\alpha \beta}}}{R^{2}}\right],
$$

where $\nu$ is a mass factor and $a_{\alpha \beta}$ is the heavy-lightscattering length. $a_{\alpha \beta}$ is related to $B_{\alpha \beta}$ by the effective range expansion $k_{0}=\left(1 / a_{\alpha \beta}\right)+(1 / 2) r_{0} k_{0}^{2}$, where $\nu B_{\alpha \beta}=k_{0}^{2}$ and $r_{0}$ is the effective range. For $B_{\alpha \beta}=0$, the potential $V(R)$ exhibits the Efimov effect, provided that the heavy-light mass ratio is big enough to make the adiabatic approximation valid. In this limit, we relate the three-body binding energy of the $(N+1)$-th Efimov state $\left(B_{3}^{(N+1)}\right)$ to the three-body binding energy of the $N$ th state. By using a scale $R^{\prime}=\sqrt{B_{3}^{(N)}} R$ and $V^{\prime}\left(R^{\prime}\right)=V(R) / B_{3}^{(N)}$, we have the following dimensionless $s$-wave Schrödinger equation $\left(\hbar=1, M_{\alpha}=1\right)$ :

$$
\left[-\frac{d^{2}}{d R^{\prime} 2}+V^{\prime}\left(R^{\prime}\right)\right] \phi=\left(\frac{B_{\alpha \beta}}{B_{3}^{(N)}}-\frac{B_{3}^{(N+1)}}{B_{3}^{(N)}}\right) \phi
$$

The differential equation (2) needs one boundary condition in the range of the two-body potential $\sim r_{0}$. The effective potential for $R^{\prime} \rightarrow 0$, if not regularized, collapses the threebody system. The boundary condition for the wave function at $r_{0}$ is a function of $B_{3}^{(N)}, B_{\alpha \beta}$, and $r_{0}: \xi\left(B_{3}^{(N)}, B_{\alpha \beta}, r_{0}\right)$. In dimensionless units, this function is written as $\xi^{\prime}\left(1, B_{\alpha \beta} / B_{3}^{(N)}, \sqrt{B_{3}^{(N)}} r_{0}\right)$. The boundary condition is the same for the Efimov states in the limit of zero range, which implies

$$
\begin{aligned}
\lim _{r_{0} \rightarrow 0} \xi^{\prime}\left(1, \frac{B_{\alpha \beta}}{B_{3}^{(N)}}, \sqrt{B_{3}^{(N)}} r_{0}\right) \\
=\lim _{r_{0} \rightarrow 0} \xi^{\prime}\left(\frac{B_{3}^{(N+1)}}{B_{3}^{(N)}}, \frac{B_{\alpha \beta}}{B_{3}^{(N)}}, \sqrt{B_{3}^{(N)}} r_{0}\right) .
\end{aligned}
$$

Consequently, $B_{3}^{(N+1)} / B_{3}^{(N)}$ is a function of $B_{\alpha \beta} / B_{3}^{(N)}$ for $r_{0}=0$. This illustrates that it is reasonable to expect a universal scaling function connecting $B_{3}^{(N+1)} / B_{3}^{(N)}$ and $B_{\alpha \beta} / B_{3}^{(N)}$ for $N \rightarrow \infty$, while the ratio $B_{\alpha \beta} / B_{3}^{(N)}$ is kept fixed. Such a scaling function is calculated in the RZR model, and we found that it converges fast with $N$.

Now, we return to our general case of three particles, $\alpha-\alpha-\beta$, to obtain the universal scaling function. We first distinguish the bound $(+)$ or virtual $(-)$ states by

$$
K_{\alpha \gamma} \equiv \pm \sqrt{B_{\alpha \gamma}}=\frac{1}{\sqrt{2 \mu_{\alpha \gamma}} a_{\alpha \gamma}}
$$

where $\gamma=\alpha, \beta$ and $\mu_{\alpha \gamma}$ is the corresponding reduced mass of the $(\alpha \gamma)$ system. The scaling function, defined by

$$
\frac{B_{3}^{(N+1)}}{B_{3}^{(N)}}=F\left(\frac{K_{\alpha \alpha}}{\sqrt{B_{3}^{(N)}}} ; \frac{K_{\alpha \beta}}{\sqrt{B_{3}^{(N)}}} ; A\right),
$$

where $A \equiv M_{\beta} / M_{\alpha}$, gives the ratio of the energies of two consecutive states $(N$ and $N+1)$ in the limit of $N \rightarrow \infty$.

Next, we present a single integral equation, which corresponds to the reduction of the coupled Faddeev integral equations [9] for the zero-range limit of a two-body interaction. To solve the integral equation, we used a regularization parameter $\Lambda$ in the momentum integration, which represents the inverse of the interaction range [3,9]. Later on, $\Lambda \rightarrow \infty$ is considered, while the ratios between each of the two-body energies, $B_{\alpha \gamma}$, and the three-body ground-state energy are kept fixed. This limit is achieved by making $N \rightarrow \infty$ with fixed $\Lambda$. The $s$-wave projected equation is given by

$$
\begin{aligned}
\chi_{\alpha \beta}(q)= & \tau_{\alpha \beta}\left(q ; B_{3}^{(N)}\right) \int_{0}^{\Lambda} d k\left[A G_{\beta}\left(q, k ; B_{3}^{(N)}\right)\right. \\
& +2 \int_{0}^{\Lambda} d p G_{\alpha}\left(p, q ; B_{3}^{(N)}\right) \\
& \left.\times \tau_{\alpha \alpha}\left(p ; B_{3}^{(N)}\right) G_{\alpha}\left(p, k ; B_{3}^{(N)}\right)\right] \chi_{\alpha \beta}(k)
\end{aligned}
$$

where, for $\gamma=\alpha, \beta$ with $\eta_{\gamma} \equiv M_{\alpha} /\left(2 \mu_{\alpha \gamma}\right)$,

$$
\begin{gathered}
G_{\gamma}(q, k ; E) \equiv \ln \frac{E-q k+\eta_{\gamma}(q+k)^{2}+q^{2}\left(\eta_{\beta}-\eta_{\gamma}\right)}{E+q k+\eta_{\gamma}(q-k)^{2}+q^{2}\left(\eta_{\beta}-\eta_{\gamma}\right)} \\
\tau_{\alpha \gamma}(q ; E) \equiv \frac{\eta_{\gamma}^{3 / 2}}{\pi}\left[\sqrt{E+\frac{A+2}{4 A \eta_{\gamma}} q^{2}}-K_{\alpha \gamma}\right]^{-1}
\end{gathered}
$$

We solve Eqs. (6)-(8) in units, such that $\Lambda=1$. The corresponding dimensionless quantities are $\epsilon_{3}^{(N)} \equiv B_{3}^{(N)} / \Lambda^{2}, \kappa_{\alpha \gamma}$ $\equiv K_{\alpha \gamma} / \Lambda$. The two-body observables can be written in terms of the three-body binding energy $B_{3}^{(N)}$, by replacing $\Lambda$, such that $\kappa_{\alpha \gamma} / \sqrt{\epsilon_{3}^{(N)}}=K_{\alpha \gamma} / \sqrt{B_{3}^{(N)}}$. The Thomas effect occurs for $\Lambda \rightarrow \infty$ with the energies of the two-body systems kept fixed, whereas the Efimov states arise when $K_{\alpha \gamma} \rightarrow 0$ with $\Lambda$ kept fixed.

In the strict Efimov limit the scaling function is a function of only $A$ and $B_{3}^{(N+1)} / B_{3}^{(N)}=F(0 ; 0 ; A)$. The ratio between 


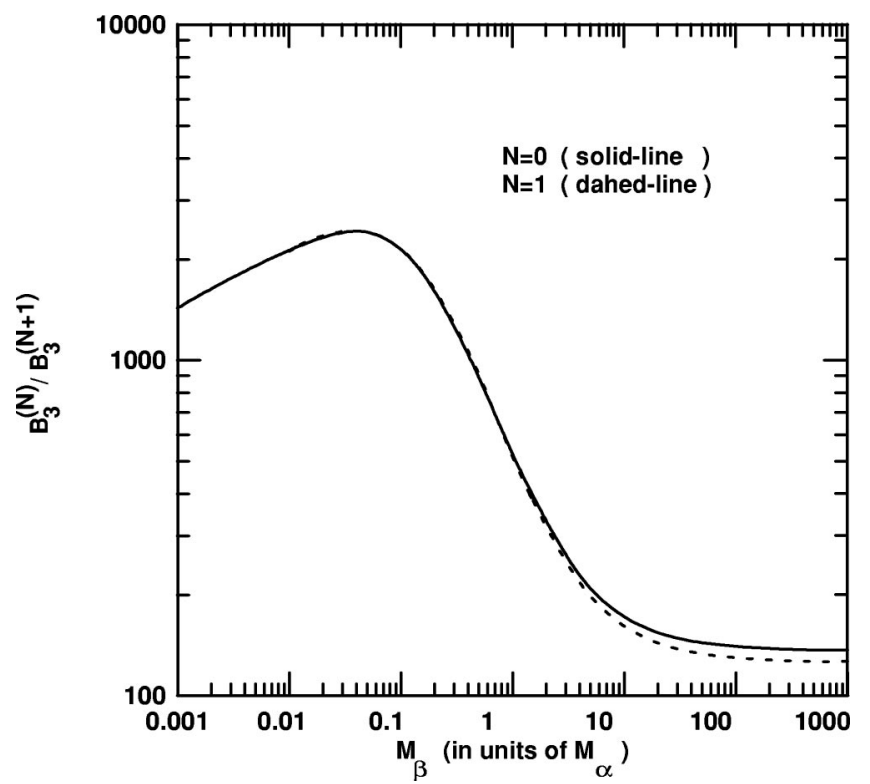

FIG. 1. The ratio between the binding energies of consecutive states in the Efimov limit ( $\left.B_{\alpha \alpha}=B_{\alpha \beta}=0\right)$, for the $\alpha-\alpha-\beta$ system, as a function of $M_{\beta}$.

two closed Efimov states becomes practically constant with $N$, as we illustrate in Fig. 1 for $N=0$ and $N=1$. For equal masses, as shown, the ratio is close to $1 / 500$.

Let us discuss the scaling function for three helium atoms. As $\beta=\alpha$ and $A=1$, Eq. (5) can be simplified to $B_{3}^{(N+1)} / B_{3}^{(N)}=F\left(K_{2} / \sqrt{B_{3}^{(N)}}\right) \quad(2$ replaces the double $\alpha$ indices). In Fig. 2, the solid line presents our result for the scaling function, which shows $\sqrt{\left(B_{3}^{(N+1)}-B_{2}\right) / B_{3}^{(N)}}$ as a function of $K_{2} / \sqrt{B_{3}^{(N)}} \equiv 1 /\left[a_{2} \sqrt{B_{3}^{(N)}}\right]$. As seen in Fig. 2, the

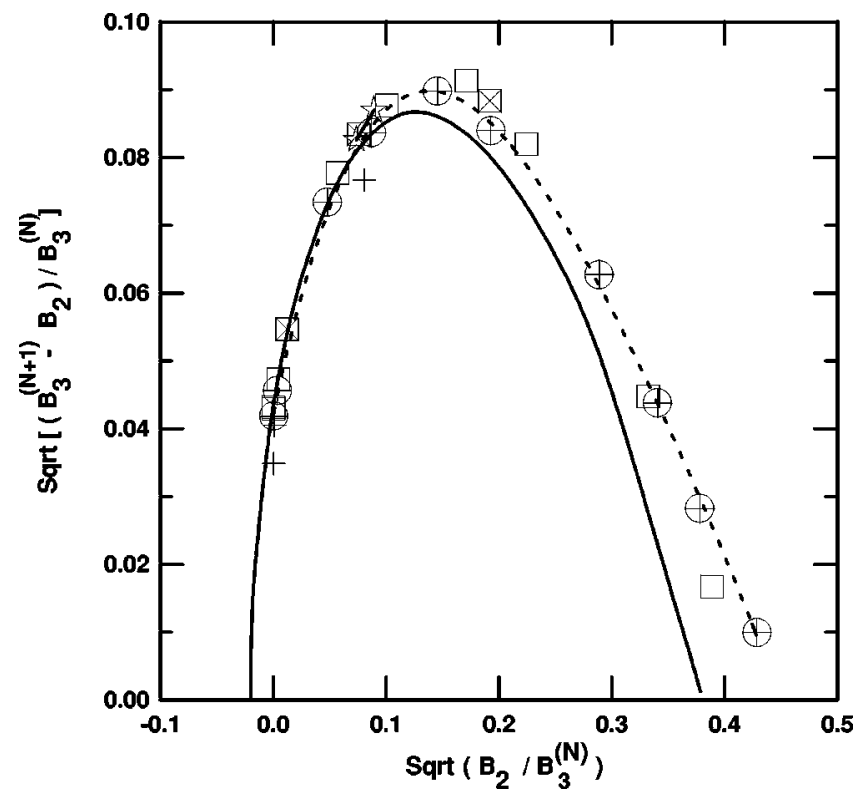

FIG. 2. Comparison of the scaling limit (solid line) with realistic calculations for the ${ }^{4} \mathrm{He}$ trimer. Results from Ref. [19] for $N=0$ for an interaction in the $s$ wave (empty boxes) and in the $s+d$ waves (crossed circles), and for $N=1$ (crosses). Other calculations for $N$ =0: Ref. [18] (stars), Ref. [20] (crossed boxes). The dashed line guides the eyes through realistic model results. Recent results from Ref. [21] are consistent with this plot.

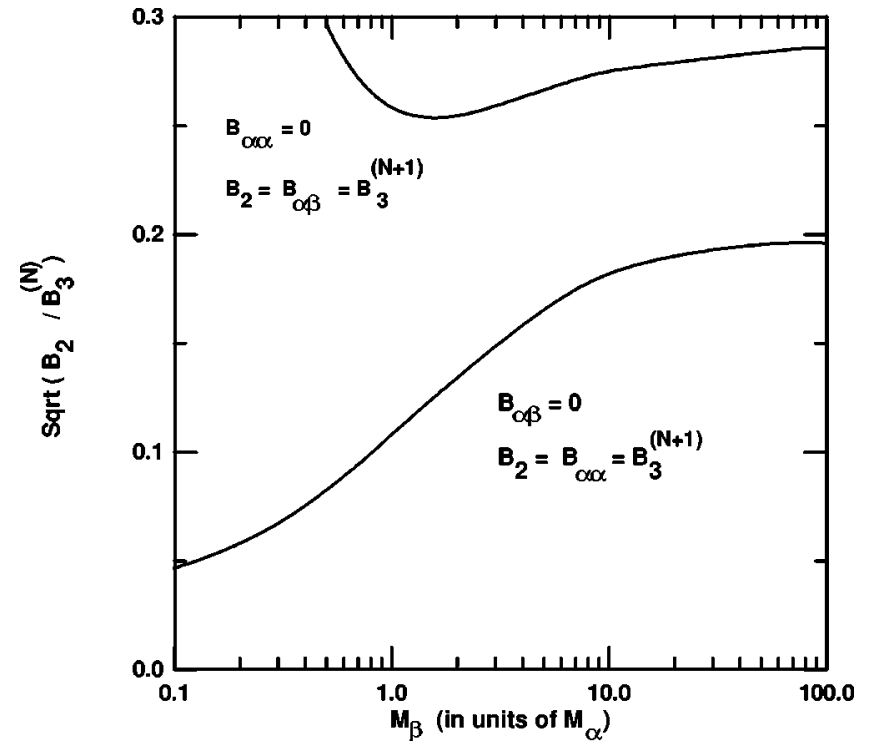

FIG. 3. Critical upper limits for the existence of one Efimov state above the $N$ th state, as a function of $M_{\beta}$.

results from realistic interactions (dashed line) approach the scaling limit. The results obtained from Ref. [19], represented in Fig. 2 by empty squares ( $s$ wave) and crossed circles ( $s+d$ waves), covered a wide range of scattering lengths, as the strength of the realistic two-body interaction was varied. So, they are appropriate for comparison with our results. We confirm that all other recent realistic calculations for the helium trimer, given in Refs. [18,20,21], are completely consistent with the scaling limit, and we show a few of them in Fig. 2.

The universality of the scaling function is a characteristic of the Efimov regime, which does not depend on the detailed nature of the short-range two-body interaction. As the twobody binding energy is increased, a small deviation between the scaling limit and realistic models can be observed. By including the effective range effect, one could improve the scaling function without requiring further details about the two-body interaction.

We have extended the analysis of the scaling function for $\alpha \neq \beta$ in two examples. The critical condition for the appearance of one Efimov state, in Eq. (5), is defined by $F\left(0 ; K_{\alpha \beta} / \sqrt{B_{3}^{(N)}} ; A\right)=B_{\alpha \beta} / B_{3}^{(N)}$, when $K_{\alpha \alpha}=0$, and by $F\left(K_{\alpha \alpha} / \sqrt{B_{3}^{(N)}} ; 0 ; A\right)=B_{\alpha \alpha} / B_{3}^{(N)}$, when $K_{\alpha \beta}=0$. For any value of $B_{\alpha \alpha} / B_{3}^{(N)}$ or $B_{\alpha \beta} / B_{3}^{(N)}$ smaller than the corresponding critical value, there exists at least one Efimov state above the ground state. As we can observe in Fig. 3, the most favorable situation, between these two, for the experimental observation of Efimov states is given when the splitting between the states becomes larger, which happens for $B_{\alpha \alpha}$ $<B_{\alpha \beta}$. These results extend the calculations given in Ref. [9] to cases where $A<1$.

Applying the results for the scaling function, obtained in Fig. 2 to ${ }^{87} \mathrm{Rb}$ atoms, we can estimate the minimum energy for the ground state, which allows for the existence of one excited Efimov state. We use only the scattering length of the ${ }^{87} \mathrm{Rb}-{ }^{87} \mathrm{Rb}$ system, $a_{R b}=50 \AA$ [24], with the assumption that it is much greater than the range of the potential, in order to estimate that $B_{2, R b}=\hbar^{2} / M_{R b} / a_{R b}^{2} \approx 0.22 \mathrm{mK}$. From the 
scaling plot presented in Fig. 2, the condition for the existence of one Efimov state above the ground state is given by $0<\sqrt{B_{2, R b} / B_{3, R b}^{(0)}} \leqslant 0.38$, since the rubidium dimer is bound. This implies that $B_{3, R b}^{(0)} \geqslant 1.5 \mathrm{mK}$, for the existence of the excited state. From this example, we show that the estimate for the Efimov-state energy demands knowledge of the ground-state energy, which could in principle be obtained experimentally; therefore, we just present the lower bound. In the same way, one can obtain the lower bound for the ground-state binding energy, which permits one Efimov state for any other system of three identical particles.

In summary, we have shown that the ${ }^{4} \mathrm{He}$-trimer ground and excited states approach the scaling limit obtained from a calculation with the renormalized zero-range model. As an example, we estimate the lower bound of the ground-state energy of the ${ }^{87} \mathrm{Rb}$ trimer, which allows one excited Efimov state, opening the possibility of studying other atomic systems. The critical conditions for the appearance of one Efimov state in the scaling limit were studied for several masses of systems with two like atoms plus a third one, for a wide range of atom-atom scattering lengths.

Our thanks to Professor R. Donangelo, Professor P.L. Ferreira, and Professor C.L. Lima for a previous helpful discussion. We also thank Professor Y. Nogami, Professor R.A. Rego, and Professor D. Sprung for their comments and a critical reading of the manuscript. This work was partially supported by Fundação de Amparo à Pesquisa do Estado de São Paulo and Conselho Nacional de Desenvolvimento Científico e Tecnológico.
[1] F. Luo, G.C. McBane, G. Kim, C.F. Giese, and W.R. Gentry, J. Chem. Phys. 98, 3564 (1993); F. Luo, C.F. Giese, and W.R. Gentry, ibid. 104, 1151 (1996).

[2] W. Schöllkopf and J.P. Toennies, Science 266, 1345 (1994); J. Chem. Phys. 104, 1155 (1996); K.T. Tang, J.P. Toennies, and C.L. Liu, Phys. Rev. Lett. 74, 1546 (1995).

[3] V. Efimov, Phys. Lett. 33B, 563 (1970); Comments Nucl. Part. Phys. 19, 271 (1990).

[4] B.D. Esry, C.H. Greene, Y. Zhou, and C.D. Lin, J. Phys. B 29, L51 (1996).

[5] C. Josserand and S. Rica, Phys. Rev. Lett. 78, 1215 (1997).

[6] P.O. Fedichev, M.N. Reynolds, and G.V. Shlyapnikov, Phys. Rev. Lett. 77, 2921 (1996).

[7] L.H. Thomas, Phys. Rev. 47, 903 (1935).

[8] S.K. Adhikari, A. Delfino, T. Frederico, I.D. Goldman, and L. Tomio, Phys. Rev. A 37, 3666 (1988); 47, 1093 (1993).

[9] A.E.A. Amorim, L. Tomio, and T. Frederico, Phys. Rev. C 56, R2378 (1997).

[10] E.J. Heller, Phys. Rev. Lett. 77, 4122 (1996).

[11] Yu. Kagan, G.V. Shlyapnikov, and J.T.M. Walraven, Phys. Rev. Lett. 76, 2670 (1996); Yu. Kagan, E.L. Surkov, and G.V. Shlyapnikov, ibid. 79, 2604 (1997).

[12] R.D. Amado and J.V. Noble, Phys. Lett. 35B, 25 (1971); Phys. Rev. D 5, 1992 (1972).
[13] A.C. Fonseca, E.F. Redish, and P.E. Shanley, Nucl. Phys. A 320, 273 (1979).

[14] S.K. Adhikari and L. Tomio, Phys. Rev. C 26, 83 (1982); S.K. Adhikari, A.C. Fonseca, and L. Tomio, ibid. 26, 77 (1982).

[15] H.S. Huber, T.K. Lim, and D.H. Feng, Phys. Rev. C 18, 1534 (1978).

[16] T.K. Lim, K. Duffy, and W.C. Damert, Phys. Rev. Lett. 38, 341 (1977).

[17] H.S. Huber and T.K. Lim, J. Chem. Phys. 68, 1006 (1978).

[18] S. Huber, Phys. Rev. A 31, 3981 (1985).

[19] Th. Cornelius and W. Glöckle, J. Chem. Phys. 85, 1 (1986).

[20] B.D. Esry, C.D. Lin, and C.H. Greene, Phys. Rev. A 54, 394 (1996).

[21] E.A. Kolganova, A.K. Motovilov, and S.A. Sofianos, Phys. Rev. A 56, R1686 (1997).

[22] S.K. Adhikari, T. Frederico, and I.D. Goldman, Phys. Rev. Lett. 74, 487 (1995); S.K. Adhikari and T. Frederico, ibid. 74, 4572 (1995).

[23] H.M.J.M. Boesten, C.C. Tsai, B.J. Verhaar, and D.J. Heinzen, Phys. Rev. Lett. 77, 5194 (1996).

[24] G. Baym and C.J. Pethick, Phys. Rev. Lett. 76, 6 (1996); J.R. Gardner et al., ibid. 74, 3764 (1995). 